\title{
Active Reperfusion Hemorrhage during Thrombectomy: Angiographic Findings and Real-Time Correlation with the CT "Spot Sign"
}

\author{
Diogo C. Haussen ${ }^{a} \quad$ Ivan M. Ferreira $^{a} \quad$ Clara Barreira $^{a}$ \\ Jonathan A. Grossberg ${ }^{a}$ Francesco Diana $^{b}$ Simone Peschillo ${ }^{b}$ \\ Raul G. Nogueira ${ }^{a}$ \\ ${ }^{a}$ Emory University School of Medicine/Marcus Stroke \& Neuroscience Center - \\ Grady Memorial Hospital, Atlanta, GA, USA; ${ }^{b}$ Hospital Policlinico Umberto I/Sapienza \\ University, Rome, Italy
}

\section{Keywords}

Stroke $\cdot$ Thrombectomy $\cdot$ Hemorrhage $\cdot$ Reperfusion injury

\begin{abstract}
Introduction: Symptomatic intracranial hemorrhage represents one of the most feared complications of endovascular reperfusion. We aim to describe a series of patients that experienced immediate reperfusion injury with active intraprocedural extravasation within the territory of the deep penetrating arteries and provide real-time correlation with CT "spot sign." Methods: This was a retrospective analysis of patients that suffered reperfusion injury with active arterial extravasation during endovascular stroke treatment in two tertiary care centers. Results: Five patients were identified. Median age was 63 (58-71) years, $66 \%$ were male. Median NIHSS was 13.5 (9.5-23.0), platelet level 212,000 (142,000-235,000), baseline systolic blood pressure 152 (133-201) mm Hg, and non-contrast CT ASPECTS 7.0 (6.5-9.0). Two patients were taking aspirin and one had received intravenous thrombolysis. There were three middle cerebral artery M1, one internal carotid artery terminus, and one vertebrobasilar junction occlusion. Three patients had anterior circulation tandem occlusions. Stroke etiology was extracranial atherosclerosis $(n=2)$, intracranial atherosclerosis $(n=2)$, and cervical dissection $(n=1)$. The median time from onset to puncture was $5.5(3.9-8.6)$ h. Intravenous heparin was administered in all patients (median dose of $4,750[3,250-6,000]$ units) and intravenous abciximab in four. All tandem cases had the cervical lesion addressed first. Four lenticulostriates and one paramedian pontine artery were involved. Intraprocedural flat-panel CT was performed in four (80\%) cases and provided real-time correlation between the active contrast
\end{abstract}


extravasation and the "spot sign." The bailout included use of protamine, blood pressure control, and balloon guide catheter or intracranial compliant balloon inflation plus coiling of targeted vessel. All patients had angiographic cessation of bleeding at the end of the procedure with parenchymal hemorrhage type 1 in one case and type 2 in four. Three patients had modified Rankin score of 4 and two were dead at 90 days. Conclusions: Active reperfusion hemorrhage involving perforator arteries was observed to correlate with the CT "spot sign" and to be associated with poor outcomes.

(c) 2018 S. Karger AG, Basel

\section{Introduction}

Although the pathophysiology of reperfusion hemorrhage is not completely understood, a key feature is believed to be related to brain-blood barrier (BBB) breakdown [1]. Ischemia and reperfusion generate BBB disruption by hyperemia, oxidative stress and inflammation, predisposing patients to hemorrhagic transformation [2]. We here report the first series of intraprocedural reperfusion intraparenchymal hemorrhage during endovascular treatment for large vessel occlusion strokes and provide real-time correlation between active angiographic contrast extravasation and the CT "spot sign."

\section{Methods}

This was a retrospective analysis of consecutive patients that suffered immediate reperfusion injury with active perforator artery extravasation during endovascular treatment for large vessel occlusion acute ischemic stroke in two tertiary care centers. Intraprocedural flat-panel CT (Allura Xper FD20/20 biplane neuro X-ray system, Philips, The Netherlands) was performed in four cases during the period of active contrast extravasation on angiography. Spot sign was defined by (1) a focus of contrast pooling within the intracerebral hemorrhage, (2) CT density of $\geq 120$ Hounsfield units, (3) discontinuous from a blood vessel adjacent to the hematoma [3, 4], and (4) angiographic images ruling out micro-arteriovenous malformation, Moyamoya disease/syndrome, arteriovenous fistula. Spot sign mimics such as tumors and choroidal plexus had to be ruled out by follow-up imaging. Continuous variables are reported as median (IQR). Categorical variables are reported as proportions. The local Institutional Review Board waived the need for patient consent and approved the study.

\section{Results}

Five patients were identified. Baseline characteristics are described in Table 1. In summary, median age was 63 (58-71) years, 66\% were male, median NIHSS was 13.5 (9.523.0), platelet level 212,000 (142,000-235,000), baseline systolic blood pressure 152 (133201) $\mathrm{mm} \mathrm{Hg}$, and non-contrast CT ASPECTS 7.0 (6.5-9.0). Two patients were taking aspirin and none was under anticoagulation. One patient had received intravenous (IV) thrombolysis (and another patient received only 10\% of the total alteplase dose). There were three middle cerebral artery M1, one internal carotid artery terminus, and one vertebrobasilar junction occlusion. Three patients had anterior circulation tandem occlusions. Stroke etiology was extracranial atherosclerosis $(n=2)$, intracranial atherosclerosis $(n=2)$, and cervical dissection $(n=1)$.

The detailed procedural variables are shown in Table 2 . The median time from onset to puncture was 5.5 (3.9-8.6) h. IV heparin was administered in all patients for a median dose of $4,750(3,250-6,000)$ units and four patients received additional IV abciximab. The proce- 
Haussen et al.: Active Reperfusion Hemorrhage during Thrombectomy: Angiographic Findings and Real-Time Correlation with the CT "Spot Sign"

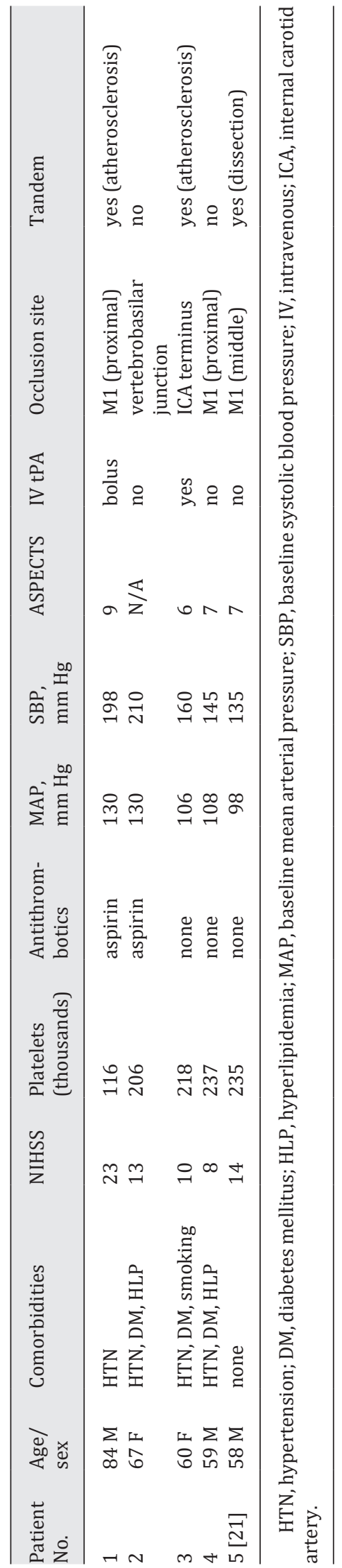
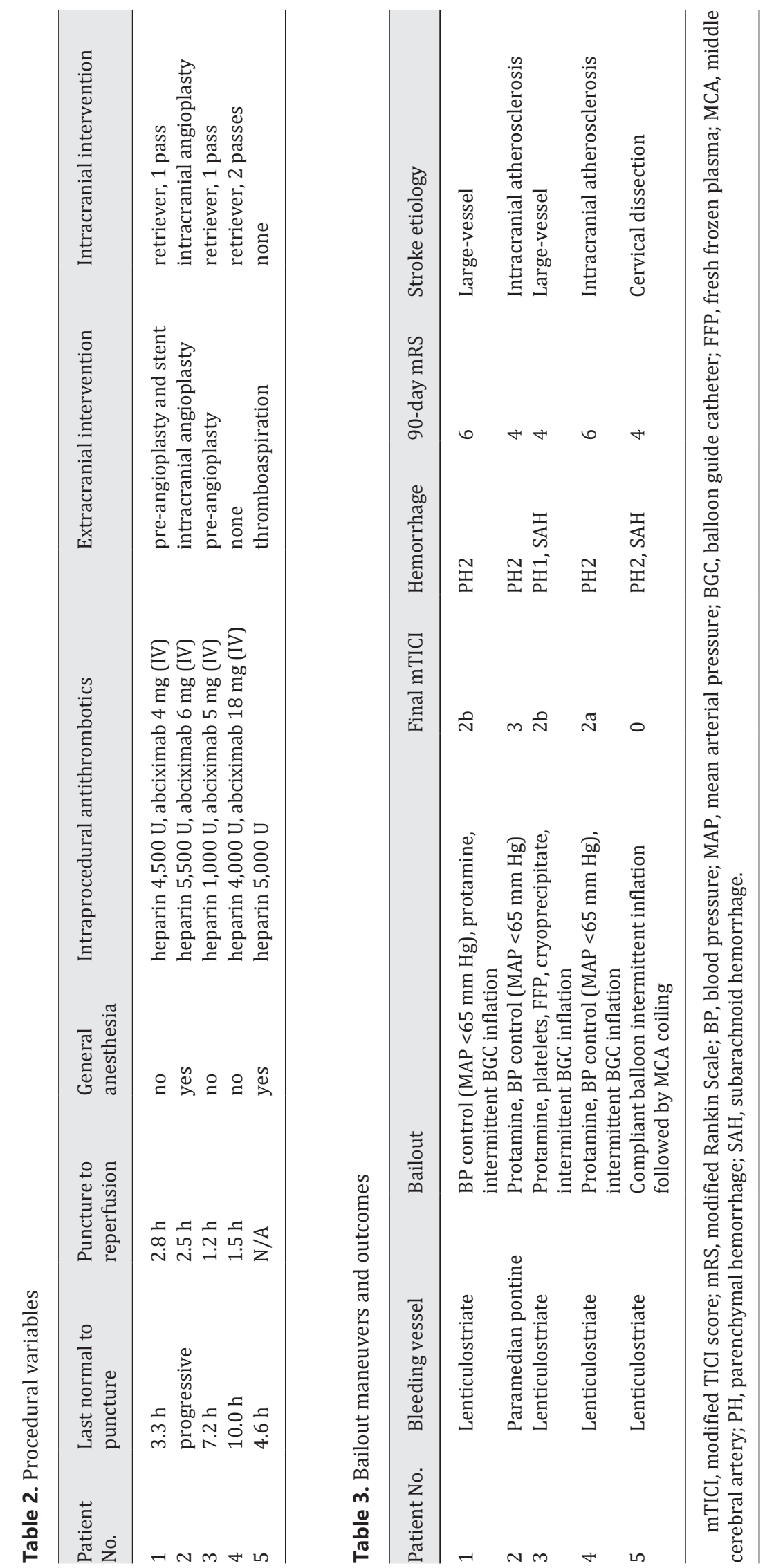

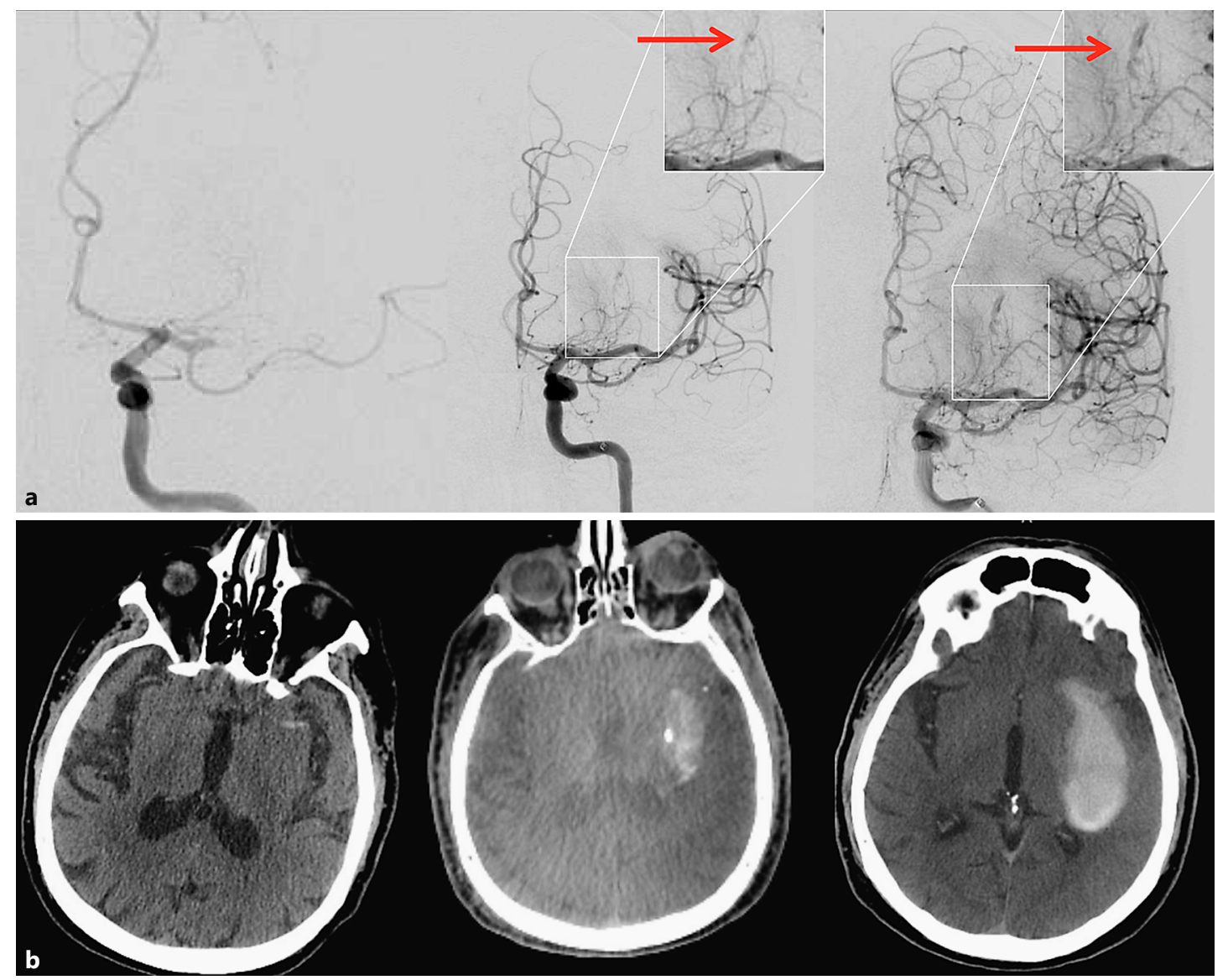

Fig. 1. Illustrative case - patient No. 1. a Left: baseline AP left internal carotid angiogram revealing an M1 occlusion; middle: early arterial phase of angiography immediately post reperfusion - arrow demonstrates the area of distal lenticulostriate extravasation; right: late arterial phase depicting the contrast extravasation. b Left: baseline non-contrast CT scan; middle: intraprocedural flat-panel CT demonstrating a "spot sign" matching the extravasation on angiography; right: 24-hour CT showing hemorrhage expansion.

dural course in all cases with tandem occlusions involved the treating the cervical lesion first. In no cases the wire was observed to be extraluminal and in all cases the site of contrast extravasation was remote from the catheterized sites. The angiographic findings of active hemorrhage were all subtle, involved the distal aspect of a perforator, and were documented in the first angiographic run after reperfusion (Fig. 1, Fig. 2, Fig. 3; online suppl. video, see www.karger.com/doi/10.1159/000488084). A flat-panel intraprocedural CT demonstrated a spot sign in all patients, which corresponded topographically to the perforating artery with active extravasation on angiography.

The bailout maneuvers and radiological and clinical outcomes are described in Table 3. Four lenticulostriates and one paramedian pontine artery were involved. The bailout included use of protamine, blood pressure control, intermittent inflation of the balloon guide catheter in the cervical internal carotid, or a compliant balloon plus coiling of targeted vessel. All patients had resolution of active extravasation by the end of the angiographic procedure. Parenchymal hemorrhage type 1 was observed in one case and type 2 in four on follow-up imaging. Only one patient (Fig. 1) expanded the hemorrhage on follow-up scan. Three patients had modified Rankin score of 4 and two were dead at 90 days. 


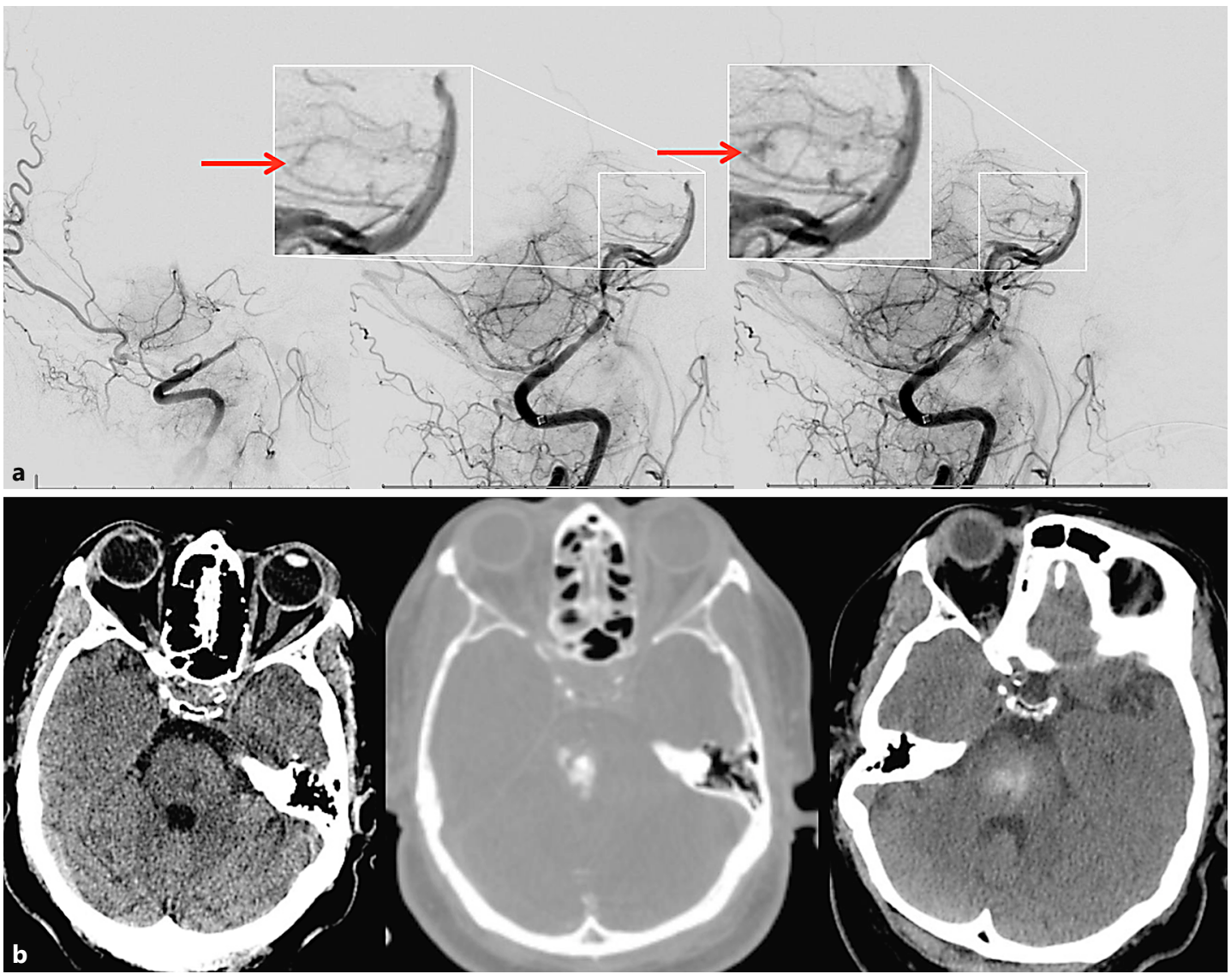

Fig. 2. Illustrative case - patient No. 2. a Left: baseline lateral vertebral angiogram revealing a distal vertebral occlusion; middle: early arterial phase of angiography immediately post reperfusion - arrow demonstrates the area of distal perforator extravasation; right: late arterial phase depicting the contrast extravasation. b Left: baseline non-contrast CT scan; middle: intraprocedural flat-panel CT demonstrating a "spot sign" matching the extravasation on angiography; right: 24-hour CT showing a small hemorrhage.

\section{Discussion}

We report five cases of active reperfusion hemorrhage during endovascular treatment. All events involved perforator arteries and were documented with angiography with concomitant flat-panel CT imaging in four cases. Despite achieving complete bleeding control, outcomes were poor.

The "spot sign" was described nearly 20 years ago as a small area of high-density material on CTA within the intraparenchymal hematoma $[5,6]$. It has been suggested to indicate extravasation of blood intermixed with contrast within the hematoma, and demonstrated to predict hematoma expansion in patients with intracerebral hemorrhage [3, 7]. To our knowledge, this is the first report of active extravasation of perforators by conventional angiography with real-time demonstration of the "spot sign," which validates the long-standing belief that this sign reflects ongoing bleeding.

The reperfusion injury mechanism that most likely explains the intraoperative hemorrhage is reactive hyperemia, which is related to the impaired vasomotor reactivity/autoregulation after reperfusion. Hyperemia is an acute elevation of regional cerebral blood flow with generation of further BBB disruption $[2,8]$. This is compatible with the previously reported 
Haussen et al.: Active Reperfusion Hemorrhage during Thrombectomy: Angiographic Findings and Real-Time Correlation with the CT "Spot Sign"
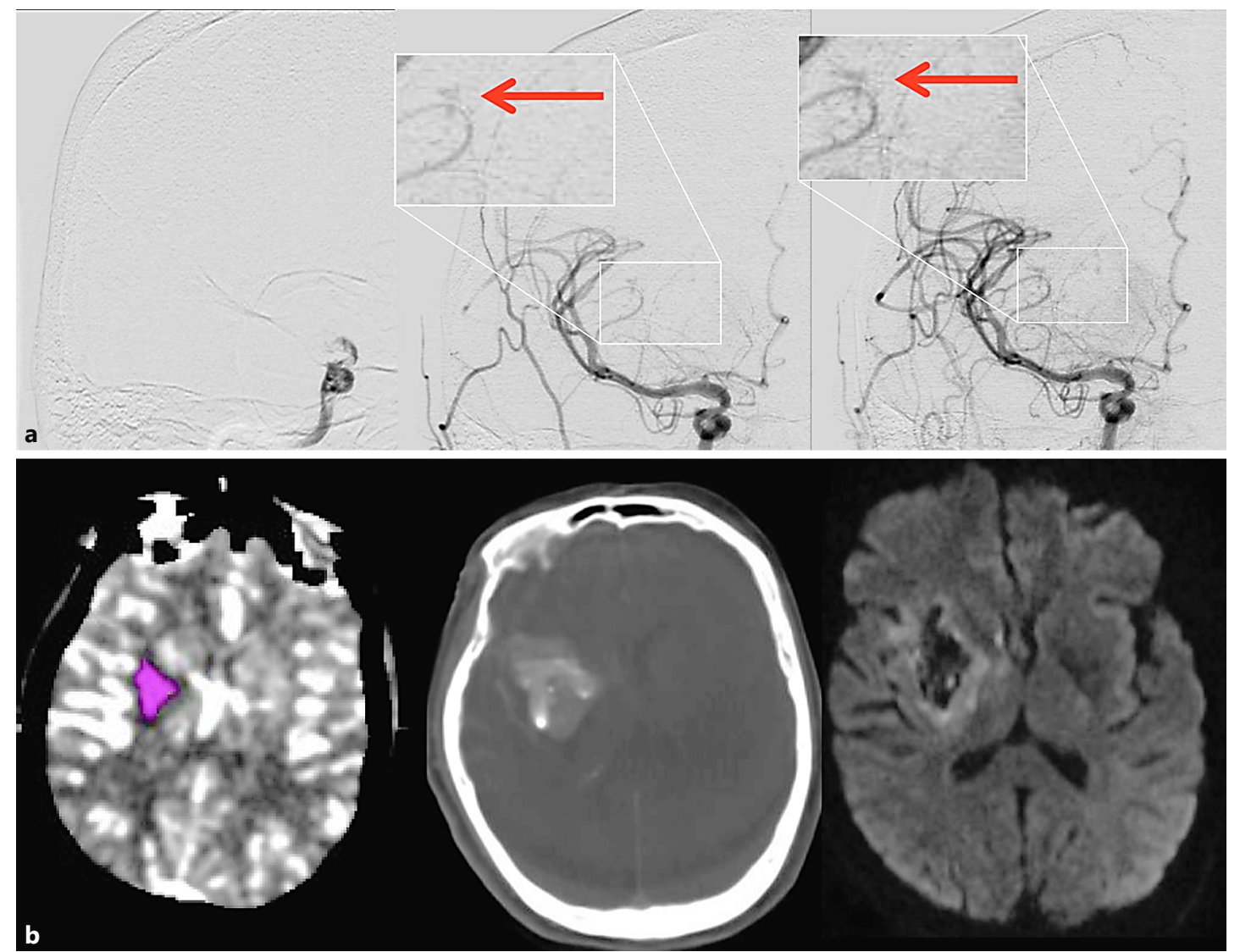

Fig. 3. Illustrative case - patient No. 3. a Left: baseline AP right internal carotid angiogram revealing an intracranial carotid occlusion; middle: early arterial phase of angiography immediately post reperfusion - arrow demonstrates the area of distal lenticulostriate extravasation; right: late arterial phase depicting the contrast extravasation. b Left: baseline CT perfusion scan showing ischemic core in pink; middle: intraprocedural flat-panel CT demonstrating a "spot sign" matching the extravasation on angiography; right: 24-hour CT showing unchanged hemorrhage volume.

findings of improved levels of reperfusion being correlated to more pronounced BBB disturbance [9].

Ischemia generates endothelial dysfunction and degradation of the basal lamina [1]. Moreover, post-ischemic reperfusion generates a surge of oxygen in the ischemic area and enhances the production of free radicals and release of proteases from endothelial cells, astrocytes, microglia, and neurons, which further aggravates the injury to the neurovascular unit [1]. The oxidative stress has been described to develop 3-8 $\mathrm{h}$ post reperfusion, therefore not characterizing itself as a major variable in the currently reported intraoperative reperfusion hemorrhages [10].

Thrombectomy has been demonstrated safe (e.g., comparable rates of hemorrhagic transformation) in the setting of effective/active anticoagulation [11, 12]. A discernable shared feature of the described patients was the use of aggressive antithrombotics. The use of IV heparin during thrombectomy in association with intra-arterial thrombolysis has been associated with higher chances of intracranial hemorrhage [13]. Interestingly, heparin has been consistently described as safe during mechanical thrombectomy, even with modern stent-retriever technology [14-16]. The higher median dose of heparin in the present series 
as compared to the other studies and the use of IV glycoprotein IIbIIIa inhibitors may have contributed to the development of hemorrhage.

Controlling the blood pressure has been suggested to minimize the chances of hemorrhagic transformation after thrombectomy [17]. A $10 \mathrm{~mm} \mathrm{Hg}$ increment in maximum systolic blood pressure documented during the first $24 \mathrm{~h}$ post procedure has been independently associated with a lower likelihood of functional independence and higher odds of mortality at 3 months [18]. Therefore, aggressively controlling the blood pressure is a very reasonable action after reperfusion. Blood pressure control through IV antihypertensives was used in 3 cases of the present series as part of the bailout. The use of balloon guide catheter allowed immediate internal carotid flow arrest; the use of the balloon guide or a compliant balloon were safely used to perform intermittent inflation/deflation and attenuate the hyperemic flow response.

Thrombectomy is associated with a low risk of reperfusion hemorrhages; parenchymal hematomas type 2 were observed in $5.1 \%$ of the interventional arm as compared to $5.3 \%$ in the medical management group in a meta-analysis including five recent randomized thrombectomy trials [19]. Hemorrhages are typically caused by reperfusion injury but can also be generated by iatrogenesis (such as a microwire perforation), which is much less common and was not observed in the present series. Contrast has been speculated to potentially have a chemotoxic effect in the disrupted BBB $[2,20]$. However, the impact of this theoretical effect in immediate hemorrhage from reperfusion may not be significantly relevant. This study has limitations inherent to its retrospective nature.

\section{Conclusions}

Active reperfusion hemorrhage involving perforator arteries was observed to correlate with the CT "spot sign" and to be associated with poor outcomes despite eventual control of the bleeding.

\section{Disclosure Statement}

D.C.H., I.M.F., C.B., J.A.G., F.D., S.P.: No relevant disclosures. R.G.N.: Stryker-Neurovacular (Trevo-2 \& DAWN/Trial PI), Covidien (SWIFT \& SWIFT-PRIME/Steering Committee, STAR Trial/Core Lab), Penumbra (3-D Separator Trial/Executive Committee).

\section{References}

1 Hayakawa M: Reperfusion-related intracerebral hemorrhage. Front Neurol Neurosci 2015;37:62-77.

-2 Khatri R, McKinney AM, Swenson B, Janardhan V: Blood-brain barrier, reperfusion injury, and hemorrhagic transformation in acute ischemic stroke. Neurology 2012;79(suppl 1):S52-S57.

-3 Peng WJ, Reis C, Reis H, Zhang J, Yang J: Predictive value of CTA spot sign on hematoma expansion in intracerebral hemorrhage patients. Biomed Res Int 2017;2017:4137210.

-4 Delgado Almandoz JE, Yoo AJ, Stone MJ, Schaefer PW, Goldstein JN, Rosand J, Oleinik A, Lev MH, Gonzalez RG, Romero JM: Systematic characterization of the computed tomography angiography spot sign in primary intracerebral hemorrhage identifies patients at highest risk for hematoma expansion: the spot sign score. Stroke 2009;40:2994-3000.

5 Becker KJ, Baxter AB, Bybee HM, Tirschwell DL, Abouelsaad T, Cohen WA: Extravasation of radiographic contrast is an independent predictor of death in primary intracerebral hemorrhage. Stroke 1999;30:20252032.

6 Murai Y, Takagi R, Ikeda Y, Yamamoto Y, Teramoto A: Three-dimensional computerized tomography angiography in patients with hyperacute intracerebral hemorrhage. J Neurosurg 1999;91:424-431. 


\section{Interventional Neurology}

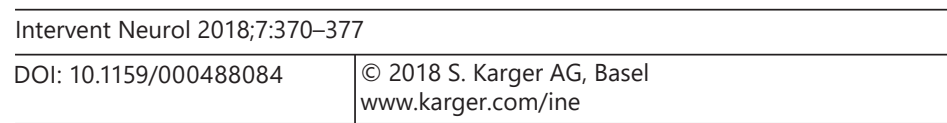

Haussen et al.: Active Reperfusion Hemorrhage during Thrombectomy: Angiographic Findings and Real-Time Correlation with the CT "Spot Sign"

7 Demchuk AM, Dowlatshahi D, Rodriguez-Luna D, Molina CA, Blas YS, Dzialowski I, Kobayashi A, Boulanger JM, Lum C, Gubitz G, Padma V, Roy J, Kase CS, Kosior J, Bhatia R, Tymchuk S, Subramaniam S, Gladstone DJ, Hill MD, Aviv RI; PREDICT/Sunnybrook ICH CTA study group: Prediction of haematoma growth and outcome in patients with intracerebral haemorrhage using the CT-angiography spot sign (PREDICT): a prospective observational study. Lancet Neurol 2012;11:307-314.

8 Nour M, Scalzo F, Liebeskind DS: Ischemia-reperfusion injury in stroke. Interv Neurol 2013;1:185-199.

-9 Yang GY, Betz AL: Reperfusion-induced injury to the blood-brain barrier after middle cerebral artery occlusion in rats. Stroke 1994;25:1658-1664; discussion 1664-1665.

10 Heo JH, Han SW, Lee SK: Free radicals as triggers of brain edema formation after stroke. Free Radic Biol Med 2005;39:51-70.

11 Rebello LC, Haussen DC, Belagaje S, Anderson A, Frankel M, Nogueira RG: Endovascular treatment for acute ischemic stroke in the setting of anticoagulation. Stroke 2015;46:3536-3539.

12 Purrucker JC, Wolf M, Haas K, Rizos T, Khan S, Dziewas R, Kleinschnitz C, Binder A, Groschel K, Hennerici MG, Lobotesis K, Poli S, Seidel G, Neumann-Haefelin T, Ringleb PA, Heuschmann PU, Veltkamp R: Safety of endovascular thrombectomy in patients receiving non-vitamin $\mathrm{K}$ antagonist oral anticoagulants. Stroke 2016;47: 1127-1130.

13 del Zoppo GJ, Higashida RT, Furlan AJ, Pessin MS, Rowley HA, Gent M: PROACT: a phase II randomized trial of recombinant pro-urokinase by direct arterial delivery in acute middle cerebral artery stroke. PROACT Investigators. Prolyse in Acute Cerebral Thromboembolism. Stroke 1998;29:4-11.

14 Farook N, Haussen D, Sur S, Snelling B, Gersey Z, Yavagal D, Peterson E: Role of heparin during endovascular therapy for acute ischemic stroke. Clin Neurol Neurosurg 2016;145:64-67.

15 Nahab F, Walker GA, Dion JE, Smith WS: Safety of periprocedural heparin in acute ischemic stroke endovascular therapy: the multi MERCI trial. J Stroke Cerebrovasc Dis 2012;21:790-793.

16 Winningham MJ, Haussen DC, Nogueira RG, Liebeskind DS, Smith WS, Lutsep HL, Jovin TG, Xiang B, Nahab F: Periprocedural Heparin Use in Acute Ischemic Stroke Endovascular Therapy: The TREVO 2 Trial. J Neurointerv Surg 2017, DOI: 10.1136/neurintsurg-2017-013441.

17 Mokin M, Kass-Hout T, Kass-Hout O, Zivadinov R, Mehta B: Blood pressure management and evolution of thrombolysis-associated intracerebral hemorrhage in acute ischemic stroke. J Stroke Cerebrovasc Dis 2012 ; 21:852-859.

18 Goyal N, Tsivgoulis G, Pandhi A, Chang JJ, Dillard K, Ishfaq MF, Nearing K, Choudhri AF, Hoit D, Alexandrov AW, Arthur AS, Elijovich L, Alexandrov AV: Blood pressure levels post mechanical thrombectomy and outcomes in large vessel occlusion strokes. Neurology 2017;89:540-547.

19 Goyal M, Menon BK, van Zwam WH, Dippel DW, Mitchell PJ, Demchuk AM, Davalos A, Majoie CB, van der Lugt A, de Miquel MA, Donnan GA, Roos YB, Bonafe A, Jahan R, Diener HC, van den Berg LA, Levy EI, Berkhemer OA, Pereira VM, Rempel J, Millan M, Davis SM, Roy D, Thornton J, Roman LS, Ribo M, Beumer D, Stouch B, Brown S, Campbell BC, van Oostenbrugge RJ, Saver JL, Hill MD, Jovin TG; HERMES collaborators: Endovascular thrombectomy after large-vessel ischaemic stroke: a meta-analysis of individual patient data from five randomised trials. Lancet 2016;387:1723-1731.

20 Yoon W, Seo JJ, Kim JK, Cho KH, Park JG, Kang HK: Contrast enhancement and contrast extravasation on computed tomography after intra-arterial thrombolysis in patients with acute ischemic stroke. Stroke 2004; 35:876-881.

21 Peschillo S, Diana F, Colonnese C, Mellina V, Marzetti F, La Rosa I, Missori P: Real-time distal, multifocal, repeated lenticulostriate bleeding points during thrombectomy in a patient with acute variable M1 occlusion: a case report and a literature review. J Stroke Cerebrovasc Dis 2017;26:2082-2086. 